\title{
A New Approach to Testing Patients in Acute Psychiatric Hospitals
}

\author{
Connor Patros ${ }^{1 *}$, and Michael Genovese ${ }^{2 *}$ \\ 'Mindyra Health Corporation, Philadelphia, Pennsylvania, USA \\ ${ }^{2}$ Acadia Healthcare, New York, USA
}

Article Info

\section{Article Notes}

Received: January 18, 2022

Accepted: February 02, 2022

\section{${ }^{*}$ Correspondence:}

Dr. Connor Patros, Mindyra Health Corporation, Philadelphia, Pennsylvania, USA

Dr. Michael Genovese, Acadia Healthcare, New York, USA

${ }^{\oplus} 2022$ Patros C \& Genovese M. This article is distributed under the terms of the Creative Commons Attribution 4.0 International License.
Accurately and systematically evaluating the mental health of patients utilizing standardized, validated measures in acute psychiatric hospitals has, for many years, presented a number of unique challenges.

First, patients in these settings often present with what are known as high severity problems, such as suicidal thoughts and behaviors, acute substance use disorders, and psychosis. In conjunction with these issues, it is not uncommon for patients to be admitted into acute psychiatric hospitals involuntarily. Amidst such high severity, complex difficulties it can become extremely challenging for individuals to accurately report on their own behavioral and emotional challenges, leading self-report surveys to be limited in their utility. As a result, acquiring valid data that can be used to quantify the degree of their pathology and progress in treatment has been challenging.

After reviewing the literature and consulting with several acute psychiatric hospital CMO's, it became apparent that there is no industry standard measurement tool to systematically track the problems and progress of acute psychiatric patients. In order to address this issue, our organizations worked together to apply a new testing system across an entire healthcare system. The assessment is known as the Clinician Observed Behaviors Scale, or COBS ${ }^{\mathrm{m}}$. It's completed by a clinician when a patient arrives at an acute care facility and when they are discharged. It includes six items that assess common acute psychiatric patient problems, and takes clinicians about 1 minute to complete. The test can be completed by any number of medical or mental health providers including physicians, nurse practitioners, nurses, psychiatrists, psychologists, therapists, social workers, and health coaches.

Working together to apply this testing system across all of Acadia Healthcare gave us a tremendous amount of data to look at how the COBS compares with alternative measurement tools, such as the Clinical Global Impressions-Severity Scale (CGI-S), that have been around for many years but do not always provide the level of detail desired when assessing a patient's overall mental health profile.

Partnering together allowed us to gather data from more than 22,000 patients across the Acadia Healthcare system. For each patient, we compared their COBS total score to their score on the CGI-S. The scores showed a high level of correlation (.78), indicating a large-magnitude, statistically-significant relationship between the two metrics. 
"So what?" you might be asking yourself at this point. If the COBS correlates so closely to a more widely-used, industry standard test, why do we need it? For one thing, those traditional testing methods do not paint the full picture of an individual patient. For example, the CGI-S focuses exclusively on patient severity using a single score, but does not tell a provider anything about specific patient difficulties. If the goal is to incorporate these data into patient care, a deeper level of detail about presenting problems is critical.

The high correlative relationship between the COBS and the CGI-S indicates that the COBS is a viable tool for evaluating patients in acute care settings. The incremental added value of the COBS is that it quantifies functioning across six common problem areas. This means it can be used to monitor individual patient improvement across more specific domains while still adhering to the broader value of a test like the CGI-S.
In our view, there is no better current alternative that clinicians are using in the acute psychiatric space. As we see more movement in the direction of having national benchmarks to better understand patient care, we believe the COBS represents an opportunity to lead that change. As our health system moves towards value-based care, a testing system like this allows us to track patient progress and puts us ahead of the pack in the acute setting. It now becomes quite straightforward to demonstrate the improvements our patients make in a more objective manner.

Beyond that, we believe it's simply the right assessment to improve clinical outcomes. We could talk for hours about data, compliance, and the importance of a value-based care model for business, but what it really comes down to is positively impacting patients. That's our main mission. If we find more effective ways to do that, we are improving on our core mission in mental health.

Connor Patros, Ph.D. is chief clinical advisor at Mindyra Health Corporation. Dr. Michael Genovese is chief medical officer at Acadia Healthcare. 